\title{
Affective Computing Applications in Distance Education
}

\author{
Jing Liu ${ }^{\mathrm{a}}$, Jun Tong ${ }^{\mathrm{b}}$, Jun Han ${ }^{\mathrm{c}}$, Fan Yang ${ }^{\mathrm{d}}$, Shuo Chen ${ }^{\mathrm{e}}$ \\ Department of Educational Technology, Capital Normal University, Beijing, 100048, China \\ aemail: fionaliujing@163.com, bemail: tongjun02.student@sina.com, cemail: \\ jameshan@vip.sina.com, ${ }^{\mathrm{d}}$ email:www.yangfan2007-47@163.com, eemail:
} cskaoyandota@sina.com

Keywords: Affective Computing; Distance Education; Lack of Emotion; Application Status

\begin{abstract}
With the rapid development of network technology, the modern distance education has become an important trend in the development of international education. However, the spatial and temporal separation of the modern distance education so that learners cannot meet the exchange of feelings and emotional missing, which would affect students' cognitive learning and psychological health. Affective computing is about emotion, and the emotional generation, as well as effect of the emotional aspects, there is still a relatively new field of study. Affective computing technology in distance education can effectively solve the problem of lack of emotional exchange of students in distance learning. This paper introduces the concept of affective computing, research content, and affective computing applications in distance education.
\end{abstract}

\section{Introduction}

Modern distance education is an important trend of international education since the 1980s, which is an important way to achieve educational information and internationalization. Modern distance education has led to a profound transformation in the mode of education and concept of education, relying on techniques and tools are very advanced, but, there are still insufficient in terms of the concept of positioning, network system and two-way interaction, especially in the two-way interaction. The spatial and temporal separation of the modern distance education so that learners cannot meet the exchange of feelings and emotional, which would affect students' cognitive learning and psychological health. Therefore, emotional compensation is of great significance for the protection of modern distance education teaching quality and the improvement of modern distance education teaching effect.

In recent years, affective computing is hot spots in information science, cognitive science and psychology of scientific research. It is based on the theory of human emotions and feelings. Its research content is emotional mechanism, access to emotional information, emotional pattern recognition, modeling and understanding of emotions, emotional synthesis and expression, emotional transfer and exchange. Its ultimate goal is to give computer similar to human emotional competence.

Affective computing focus of the study that is obtained the signal through a variety of sensors physiological and behavioral characteristics caused by human emotion, establish emotional model, thus create a perception, recognition and understanding of human emotions and to make intelligent, sensitive and friendly response for the user's emotional personal computer systems. It will help shorten the distance between man and machine, to create a harmonious human-machine environment. Affective computing applications in online education, it can give the corresponding emotional encouragement or emotional compensation strategy, according to the learner's specific emotional state, largely compensate for the lack of emotion of the modern distance education and to achieve a harmonious man-machine emotion interaction.

\section{The organization of distance learner's emotional information}

Distance education provides a convenient, fast and economical mode of teaching. However, due to the separation of teachers and students, students cannot exchange timely, dynamic, face-to-face like the conventional education. Some problems of students in the learning process are not solved in time, and their confusion can't get help in the psychological. Students are prone to isolation and loneliness, and thus lose confidence and interest in learning. And it is difficult for students to feel the teachers on their concerns, easily lead to confusion, lazy in the study. Students will cause serious 
impact, and students will learn to generate resentment [1].

At present, the lack of emotional communication in distance education has attracted more and more attention of scholars, and they has also been tried a number of solutions. Taking advantage of the network and multimedia technology to create virtual teaching situation; Build collaborative learning mode; Using the technology of interactive video, streaming media to communicate [2]. These strategies can avoid the lack of emotional communication in distance learning in a certain extent, but cannot accurately give the emotion encourage or emotional strategies corresponding according to learners specific emotional states. Therefore it cannot fundamentally help learners solve the problem of the lack of emotion. Affective computing is introduced in distance education can effectively solve this problem.

It is a complex process that distance learners organize affective information. In general, the emotion may obtain and measure through the physiological and psychological means [3]. Physiological measurement method is that, with the help of sensors and other measuring instruments, measuring people's brain waves, heartbeat, skin sap, potential, respiratory, facial expressions and other physiological changes, to understand the emotional state of people for emotional information [4]. Getting emotional information mainly use the physiological measurement in Emotional information processing and affective computing technology. Psychological measurement includes the questionnaire method, adjective check list method and projective test method, also known as subjective experience measurement method. Among them, the questionnaire surveys the current emotional state and psychological feelings by questionnaire. Semantic differential scale is one of the common means, it consists of a certain vocabulary and scale of the expression of emotional experience. Scale contrast to the two meanings of the adjective as bipolar, according to the degree of difference is equally divided into 5 to 7, it select the appropriate level to make judgments based on the emotional level of awareness by the user. It can understand the emotional experience of the user on an objective object by comprehensive the result of the judgment of the user [5].

In order to have full access to the remote learner's emotional information, it can be combined physiological measurement method with psychological measurement strategy in distance learning support system. Taking into account the special needs of distance education, on the one hand, we can understand the emotional distance learners by the network terminal questionnaire static preliminary; On the other hand, the affective computing terminal real-time monitor distance learners in the learning process, collecting distance learners facial expressions, voice emotion, attitude emotional and physiological indicators of dynamic emotional information [6]. Among them, the facial expressions and speech emotion is the key.

Combined with the characteristics of distance education, learning-related expression information can be able to identify. Therefore, compared with the general expression recognition, expression recognition is relatively easy for fewer types of distance education. For distance learners, you can define four learning expression, such as happy, bored, curious, disappointed. It calculates the parameters of facial expressions by expression modeling, tracking changes in emotional state.

It can distinguish between two types of learning interest and bored expression, using the aversion degree and the focus to describe the distance learners' mental state associated with the emotional state. The so-called aversion degree is through face detection to determine the level of interest of the learner in the learning process of the current learning content [7]. Relative to the normal state, when the detected face contour larger, it indicates that learners leaned forward and very interested in the content of the current study, the aversion degree, aversion to large. When the detected face contour becomes smaller, it indicates that learners lean back and are not interested in the content of the current study, even boredom, aversion to little. The so-called degree of focus, it determines the degree of concern of learners in the learning process of the current learning content by face detection. Relative to the normal state, when the detected face eye spacing larger, it indicates that the learners are very concerned about the current learning content, degree of focus is high. When eye spacing becomes smaller, it indicates that the learners learning lack of concentration or mental fatigue, degree of focus is low. Starting from the definition of face detection, emotional state values can be defined through training based on the degree of aversion, getting the relationship between approach-withdrawal detection and interest emotional state value, establishing the appropriate mood 
space, and then be able to judge the interest of distance learners by degree of focus. However, when the learner turned around or eyes closed (nap), based only on the degree of aversion is not correctly detect the state of interest. At this time it can be matched to focus to improve the recognition effect.

In general, facial expression recognition includes two important techniques, namely face detection and Positioning technology, as well as face image extraction and recognition technology. At present, the use of computer facial expression recognition include facial expression recognition method based on the geometric characteristics, model-based identification method and identification method based on the overall facial features. According to the actual situation of distance education, distance learners facial expression recognition generally is divided into three consecutive phases: face detection, Standardization, facial expressions classification [8]. Firstly, we get face and their organs position through the USB interface camera face detection. The image detected face and organ must pass through the image standardization to emotion recognition. Standardization is a necessary step in the emotion recognition, and it can affect the entire face emotional recognition systems. Because the input image may have different size, angle of deflection, which are adversely to emotion recognition. At this stage, that the facial feature points obtained in the first stage is control information for the face adjusted. In addition, the histogram equalization is used to balance the face image of irregular light intensity. The final stage is the classification of facial expression recognition. Through the analysis of the feature vector to represent facial features (such as pixel density) to classify facial expressions, it can produce an expression classifier.

Speech emotional processing is mainly carried out by measurement, decomposition, analysis, synthesis methods. Its purpose is to identify, understand, and synthesis component of speech signal, thereby enabling the computer to have certain voice emotion ability.

At present, classification methods are "Eight Emotion" and "Four Emotion" in the research of speech emotion recognition in the common. In distance education, it can be used four kinds of emotion, such as anger, happy, sad, fear. That emotional state at any one time is a mixture of four basic emotion, each basic emotion can be used" no, Seems to have, some, have, must have, very strong" and other state to characterize. For distance learners speech emotion recognition by computer, mainly includes emotional speech database, speech emotion feature extraction, speech emotion pattern recognition, model training.

\section{The application status of affective computing technology in distance education}

Affective computing group of the MIT media lab is the first affective computing technology applications teaching. Teaching system they developed collect data through the camera recording facial expressions and biosensor connecting in the human body, and then an "emotional assistant" adjust the program to identify emotions. If you show confusion for a piece of content on TV talk, emotional assistant will play it back or to give an explanation.

X.R. Ma put forward the "E-Learning System Based on affective computing ". The user is real-time monitoring in the learning process by affective computing terminal, and collected information about the facial expression, speech emotion passed to the affective computing server [9]. After the end of the period of study, it is the formation of the evaluation results according to the user to fill in a questionnaire and emotional result. Feedback agent evaluation results are converted into the corresponding evaluation parameters, it bring up the previous record of learning of the user from the user database, passed along to the course recommended agent. The study recommended agent accepts parameters and the previous record of learning, and adjust accordingly, user-level learning materials searched from the course materials database is passed to the interface agent. In this way, students adjust the learning progress timely according to their level, and get for their own level of learning materials.

X.W. Wang, Z.L. Wang proposed E-learning assistant system based on artificial psychology. Using Client/Server structure, the student is the client, the teacher is the server, and each student is equipped with a camera [10]. The feature of the system is the emotional information monitoring module. An important part of gesture recognition and emotion judgment is realized in this module. The video image that captured by the camera are generally students face major, according to the movement of the face, draw students learning content aversion. According to the situation and the time interval of the eye, we can know that student is napping or already asleep. Thus, we will be 
able to get the students' emotional information according to the students 'attitude change by emotion and attitude relational model. So that teachers can real-time monitor each student's emotional state to communicate like a class effect.

X.R. Ma, Z.L. Wang proposed a FPBDI Agent mental model in the "emotion interaction in distance education model "[11]. The model with a six-tuple description for FPBDI Model = (F, P, B, D, I, C), Wherein, F is the feature model for the description of the basic situation of the student / teacher; $\mathrm{P}$ is the mental model for the description of the psychological characteristics of the student / teacher; B is a model of faith, it records the students' mastery of the knowledge of the state in the student model; D is a wish model, that recorded in the student model is the goal of student learning; that recorded in the teacher's model is teaching objectives. I represent intention model, it indicates individual learning of students in student model, and it can dynamically adjust teaching strategies. Recorded class of teachers teaching strategies, it can be used as the initial intention of the students. With the students' learning activities are carried out, it will be trimmed. C is a session model, presented to the dynamic mental models, namely the display interface. FPBDI Agent mental model independently to generate appropriate emotional responses through continuously perceive changes of outside world and its state, there are certain cognitive functions. The establishment of the model is essential, for the teaching process dynamic adjustment of teaching strategies.

\section{Conclusion}

Affective computing technology is applied to the distance education system, the current is still facing many challenges. Such as emotional information acquisition and parametric modeling, to achieve human-computer interaction humane and intelligent, they need the affective computing system data together a large number of widely distributed, and then presented in a personalized way to each user. At the same time, as the application of intelligent affective network system, we also need to solve problems as follows: How to establish a more effective model to describe learning emotion expression; How to establish a more effective learning emotion model; How to design a more effective learning expression feature extraction and recognition algorithm; How to build emotional teaching model more in line with the education and psychology, etc..

\section{References}

[1] M.T. Feng, R.J. Wang, Q.Y.Ma. Affective Computing and Its Application in Network Education [J] Software Guide, Educational Technology, 2008 (4) 82-83

[2] X.L. Fu. Computer Educational Computing Electronic Learning Emotion[J]. 2004,12.

[3] H.W. Xia. Affective Computing and Its Application[R]. Human-Computer Interaction Design, Nanjing Normal University, 2008

[4] Y.W. Wu, Wei Liu, K.M. Zhang[J]. Computer Engineering and Design, Intelligent Tutoring System Based on Affective Recognition Research [2008, 29 (9): 2350- 2352

[5] J.H. Wan. Affective computing.[J] China Science and Technology Information, 2005 (15)

[6] Qi Luo, L.Y. Wan, Y.W. Wu. Application of E-learning System Exploration[J] Affective Computing. Open Education Research, 2006 (3)

[7] X.Y. Meng, Z.L. Wang, L.J. Wang. The Teaching Assistant System Modeling of Affective Computing Research [J]. Application Research of Computers, 2007 (4)

[8] G.J. Wang, Z.L.Wang, G.L.Yang. Research on Artificialemotion[J]. Application Research of Computers, 2006, (11): 26- 30

[9] X.R. Ma, Lin Liu, Jing Sang. Modeling of E-Learning System Based on Affective Computing[J]. Computer science, 2005,8,32 (8)

[10] X.W. Wang, Z.L. Wang. E-learning, Auxiliary System of Artificial Psychology Based on Microcomputer Information [J]. 2006,22(5-2)

[11] X.R. Ma, Z.L.Wang. Study on the Application of Computer Modeling of Emotion Interaction in Distance Education[J]. 2005 (9) 